\title{
Increased salt tolerance in Zymomonas mobilis strain generated by adaptative evolution
}

\author{
Katsuya Fuchino* (1D and Per Bruheim
}

\begin{abstract}
Background: Ethanologenic alphaproteobacterium Zymomonas mobilis has been acknowledged as a promising biofuel producer. There have been numerous efforts to engineer this species applicable for an industrial-scale bioethanol production. Although Z. mobilis is robustly resilient to certain abiotic stress such as ethanol, the species is known to be sensitive to saline stress at a mild concentration, which hampers its industrial use as an efficient biocatalyst. To overcome this issue, we implemented a laboratory adaptive evolution approach to obtain salt tolerant $Z$. mobilis strain.
\end{abstract}

Results: During an adaptive evolution, we biased selection by cell morphology to exclude stressed cells. The evolved strains significantly improved growth and ethanol production in the medium supplemented with $0.225 \mathrm{M} \mathrm{NaCl}$. Furthermore, comparative metabolomics revealed that the evolved strains did not accumulate prototypical osmolytes, such as proline, to counter the stress during their growth. The sequenced genomes of the studied strains suggest that the disruption of ZZ6_1149 encoding carboxyl-terminal protease was likely responsible for the improved phenotype.

Conclusions: The present work successfully generated strains able to grow and ferment glucose under the saline condition that severely perturbs parental strain physiology. Our approach to generate strains, cell shape-based diagnosis and selection, might be applicable to other kinds of strain engineering in Z. mobilis.

\section{Background}

Biofuel is a renewable clean energy source derived from biomass. With increasing environmental concerns about the use of fossil-dependent fuels, development of sustainable biofuel-refinery has lately attracted public attention. Currently, the baker's yeast Saccharomyces cerevisiae is regarded as the best promising bioethanol producer [1]. However, an alternative candidate, facultative anaerobic alphaproteobacterium Zymomonas mobilis is an efficient ethanol producer exhibiting several attractive physiological features surpassing S. cerevisiae, such as high specific rate of sugar uptake, relatively small genome size, low biomass production, a capacity to fix nitrogen gas [2$5]$, and superior ethanol productivity $[4,6]$. Its efficient homo-ethanol fermentation, mediated by the EntnerDoudoroff (ED) pathway where up to $50 \%$ of total cellular

*Correspondence: katsuyafuchino@gmail.com Department of Biotechnology and Food Science, Norwegian University of Science and Technology, Trondheim, Norway soluble protein is involved as the catalytic enzymes, coupled to active pyruvate carboxylase and two alcohol dehydrogenases, catabolizes simple sugars efficiently and produces ethanol nearly at the theoretical maximum yield in $Z$. mobilis [4]. The potential of $Z$. mobilis culture in an industrial use is not strictly restricted in converting sugars to ethanol. It has been emerging as a producer of different high-value chemicals [7-9].

To expand its potential as a biocatalyst, increasing stress tolerance is critical in actualizing Z. mobilis based biorefinery. $Z$. mobilis cells are intrinsically tolerant of very high concentration of ethanol $(>10 \%, \mathrm{v} / \mathrm{v})$ and broad $\mathrm{pH}$ range (3.5-7.5) [10]. However, the ubiquitous inorganic salt sodium chloride inhibits $Z$. mobilis growth at a mild level [10, 11]. Addition of $10 \mathrm{~g} / \mathrm{L}$ $\mathrm{NaCl}$ in the complex growth medium, a concentration commonly used in bacterial growth medium, significantly perturbed the growth and ethanol production of Z. mobilis [11]. Z. mobilis also exhibits abnormal cell shape under the salt condition, by elongating rod/oval 
shape and forming long filamentous structure with a bulged swollen pole [11]. Considering that Z. mobilis grows well in the presence of high amounts of glucose [7], osmotic pressure per se may not be the sole cause, but ionic disturbance by salt likely attributes to the poor growth.

Inorganic ions are potential inhibitors in a lignocellulosic hydrolysate, an environmentally and economically appealing substrate for biofuel production. Also, the common salt $\mathrm{NaCl}$ may be found in different ranges of renewable industrial feedstocks [12]. De-salination before fermentation is not favored due to its high costs. Thus, $Z$. mobilis strains that can grow and ferment under salt condition are in demand. Nevertheless, only few studies have tacked this problem so far [13, 14]. Previously, a systematic approach introducing transposon mutations in $Z$. mobilis identified that a mutation in $\operatorname{him} A$ increased tolerance to the saline stress [13]. Although the mechanism of action was not clarified, the himA mutant strain exhibited improved growth and ethanol production under the saline condition. The $\mathrm{Na}^{+} / \mathrm{H}^{+}$antiporter in strain ZM4 was also identified as an important transporter in the sodium ionic stress [14]. The strains obtained from these works showed promising improvements, yet, to a mild degree or under limited conditions. Interestingly, it was suggested the potential biological significance of respiratory chain-enigmatic, low-energy coupled aerobic respiration in $Z$. mobilis might be important for maintaining a low reduced/oxidized form of the co-factor Nicotinamide Adenine Dinucleotide (NAD) upon saline stress [15].

The general bacterial stress response to external high osmolality is to accumulate low molecular mass compatible solutes, through transporting and biosynthesis [16]. This type of response in Z. mobilis was previously reported, showing a relative increase of several metabolites under saline conditions [17]. On the other hand, Kohler et al. reported that $Z$. mobilis genome is missing most of the loci encoding compatible solute transport proteins such as the Kdp complex and BetS, implying that the transporting mechanism is not involved in this species to encounter saline stress [18]. In addition, accumulation of sorbitol was suggested to be important in osmotic stress response in Z. mobilis [19], however, this regulation was not confirmed in another study [15]. Taken together, these studies suggest that de novo synthesis of molecules appear to be critical for the adaptation in Z. mobilis.

For improving $Z$. mobilis cells against external abiotic stress, several studies adopted top-down or forward approaches. These includes an error-prone PCR based mutagenesis [20], an adaptive laboratory evolution method [21-23], genome shuffling [24] and a transposon approach [13].
In the present study, we improvised an adaptive laboratory evolution approach to generate saline resilient strains. The obtained strains successfully improved cellular growth and ethanol production under saline conditions. Furthermore, we examined the promising strains by quantitative metabolomics and whole genome sequencing, in order to understand what mechanism might be responsible for the salt tolerant phenotype.

\section{Results and discussion}

\section{Adaptive laboratory evolution to generate salt-resilient} strain

To generate $Z$. mobilis strains that can grow and ferment under saline stress conditions, we adopted an adaptive laboratory evolution strategy. The approach was previously employed by Wang et al., however the Z. mobilis culture with excessive $\mathrm{NaCl}$ concentration was not viable for long term due to a toxic effect, and the approach was not successful in generating resilient strain [13]. Therefore, we introduced a bias in the serial transfer to direct evolution preferable to our goal (Fig. 1).

Previously, it was shown that salt condition induces filamentation of Z. mobilis cells [11]. We observed the filamentation in our experimental setting, using our standard complex medium supplemented with $0.225 \mathrm{M}$ $\mathrm{NaCl}$ (Fig. 2). Interestingly, almost all cells exhibited an abnormal bulge at single cell pole, with variant cell length and width (Fig. 2 top right). These cells constituted a loosened pellet, found as sediment in the fully-grown culture (Fig. 1), however, we did not observe any floc formation which was shown to be beneficial for stress resistance in Z. mobilis [25]. Based on the cell shapes and the growth profile (Figs. 2,3), we speculated that the filamentous shape, especially a bulged pole, was a consequence of stress, rather than an adaptation to environment. The similar morphology of bulged filamentation was observed in the $Z$. mobilis cells growing under the hightemperature conditions $\left(39{ }^{\circ} \mathrm{C}\right)[26]$. We then thought the bulged filamentation to be exploited as a biomarker to identify the stressed cells that should be avoided for serial transfer. Although the sedimentation might have involved other factors, microscopic observations led us to conclude that filamentation of cells facilitated sediment formation.

Following this rationale, we performed lab-directed evolution with a bias in transfer. In practice, we collected cells only from an upper layer in the fully-grown culture as an inoculum for the next round (Fig. 1). The $\mathrm{Zm} 6$ strain was adapted in the complex medium supplemented with $0.2 \mathrm{M} \mathrm{NaCl}$ for 13 transfers, then continued in the medium with $0.225 \mathrm{M} \mathrm{NaCl}$ for another 8 transfers. After the first 10' about transfers, it was apparent from the turbidity of cultures that the strains 


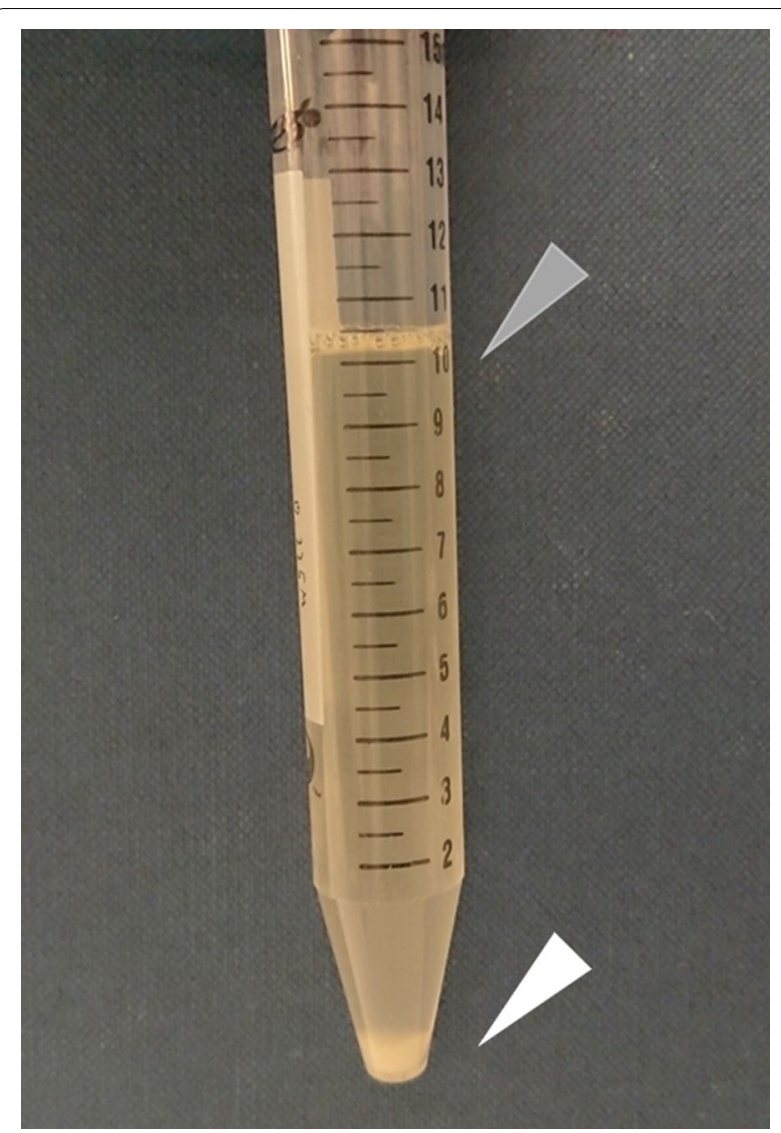

Fig. 1 Morphology-biased serial transfer for generation of salt resilient $Z$. mobilis strains. The picture shows the fully grown Zm6 culture in the complex medium supplemented with $\mathrm{NaCl}$ $0.225 \mathrm{M}$. The sediments of Zm6 Z. mobilis cells at the bottom of culture (pointed by a white arrowhead), constituting clumps of bulged/filamented cells, were observed under a phase contrast microscope. In the upper layer of the medium (pointed by a gray arrowhead), shorter filamented cells resided, and this part of culture was transferred as an inoculum for next round of evolution

demonstrated improved growth characteristics. Strains after the 13th transfer was designated as KFS1 and after the 21st transfer as KFS2. To clarify if the improved growth was due to temporal physiological adaptation without genetic mutation or whether the stable phenotype arose from mutations, we performed a pilot experiment. The evolved strain culture without adaption, i.e., grown in the medium without additional salt, was used as an inoculum for measuring the growth profile under salt condition $(0.225 \mathrm{M} \mathrm{NaCl})$. We observed the improved growth, excluding the possibility of transient adaptation and showing that mutation in the genome was the cause.

The evolved strains exhibited two characteristics in cell shape under the salt condition. The strains less frequently formed bulged pole than the parental strain did (Fig. 2, bottom panels), indicating that the evolved cells were less stressed under the salt condition. The evolved cells were also found fragmented or long filament shaped (Fig. 2).

\section{Characterization of evolved strains}

Next, we characterized phenotypes of presumably evolved strains by determining the growth curve, glucose consumption and ethanol production of the evolved and the parental strains in the presence of various salt concentrations. It should be noted that the used inoculum for the culture was not adapted to salt condition. (See Materials and methods).

As shown in the Fig. 3, the parental strain $\mathrm{Zm} 6$ did not consume all available glucose in the medium with salt, due to an arrest of growth. In sharp contrast, the evolved strains exhibited improved growth and ethanol production under the salt condition, compared to those by the parental strain under the same condition (Fig. 3). Remarkably, the final biomass of evolved strain was about 2.5 and 2.7 times higher than that of $\mathrm{Zm6}$, respectively for KFS1 and KFS2 (Fig. 3). The total ethanol production by KFS1 and KFS2 was also significantly improved, 2.74 and 2.69 times higher than by the parental strain (Fig. 3). It should be noted that the final biomass (mg.mL $\mathrm{m}^{-1}$ ) and the ethanol yield $[\mathrm{EtOH}(\mathrm{g}) / \mathrm{Glucose}(\mathrm{g})]$ by the evolved strains under the salt condition was close to those by the parental strain under the non-salt condition.

To characterize the strains further, growth profiles of all strains in the medium without supplement of salt were recorded (Fig. 3). Both evolved strains showed slightly retarded growth and ethanol production under nonsalt condition, and their final biomass was significantly lower than that of the parental strain. This was somewhat expected by us, considering that the improvement of growth in salt medium was drastic and likely involved a physiological alternation. However, the final ethanol production by all strains was nearly same (Fig. 3), showing that the final ethanol production per cell dry weight was, interestingly, higher in the evolved strains. This implies that the enzymes responsible for fermentation was more dense in the evolved strains than the parental strain. Along with this line, we observed that the evolved cells exhibited smaller cell size than the parental strain, as shown by light microscopic images (Fig. 4).

\section{Quantitative metabolomics}

The bacterial osmotic response generally involves an accumulation of osmoprotectant to counter an external osmolality, to maintain turgor. Proline and betaine-glycine are examples of well-characterized osmoprotectant in several bacteria [27]. Such a response, accumulating specific compounds as osmolytes, may result in dynamic 

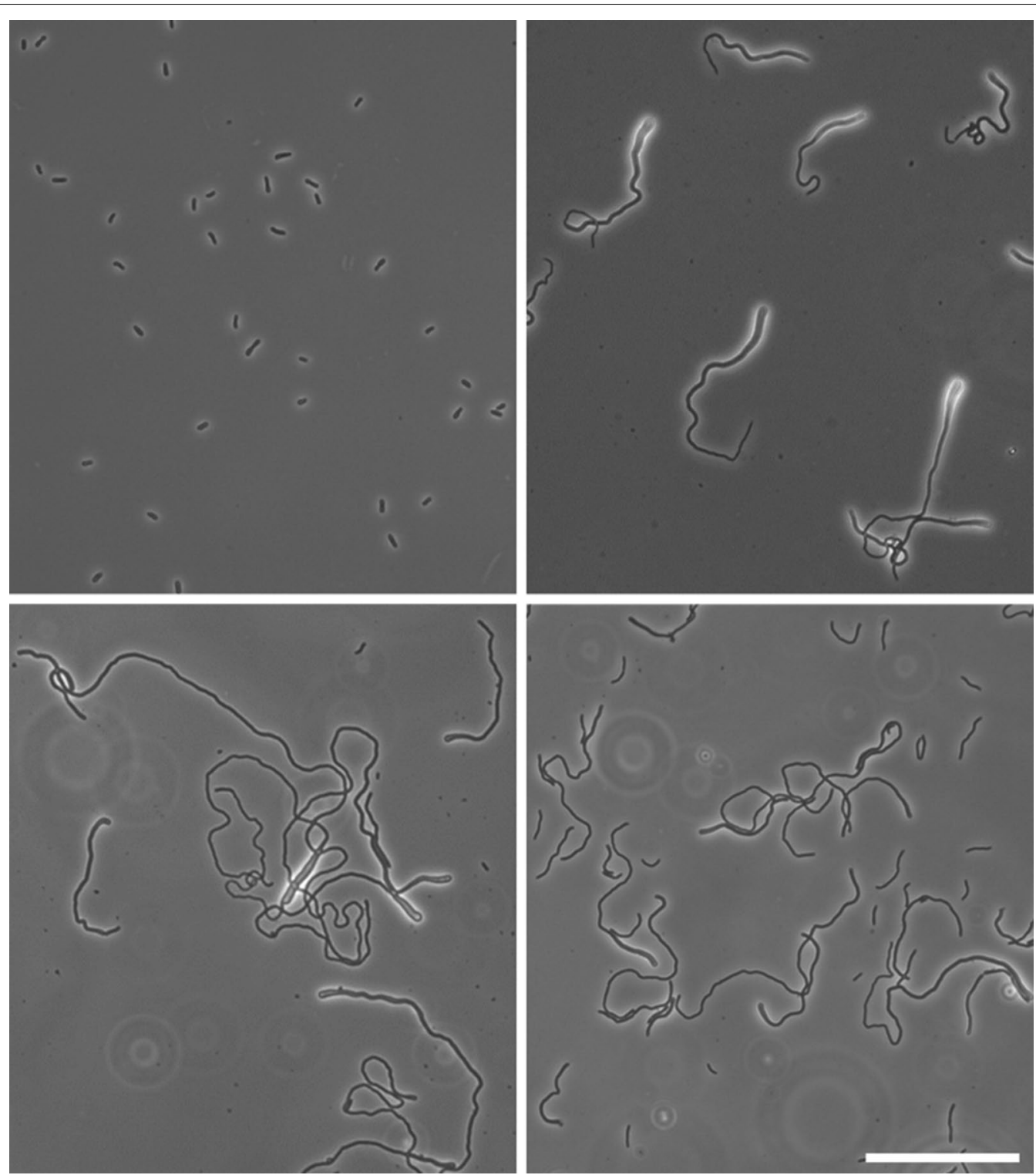

Fig. 2 Improved morphology of evolved Z. mobilis strains under salt conditions. All images were taken by a phase contrast microscope. Left top; growing Zm6 in the complex medium without addition of salt. Right top; growing Zm6 in the medium containing $0.225 \mathrm{M} \mathrm{NaCl}$, showing abnormal bulged shape at a pole of elongated cells. Left bottom; growing strain KFS1 in the complex medium with $0.225 \mathrm{M} \mathrm{NaCl}$, showing long filamentation of cells with occasional bulged shapes. Right bottom; growing strain KFS2 in the complex medium with $0.225 \mathrm{M} \mathrm{NaCl}$, exhibiting shorter filamentation and less occurrence of bulged cell pole comparing to KFS1. Scale bar applies to all images; $50 \mu \mathrm{m}$

metabolic changes and could perturb production of desirable compounds by $Z$. mobilis. To determine if any metabolomic adjustments played a significant role in the acquired resilience in the evolved strains, we performed quantitative targeted metabolic profiling of central carbon metabolites and free amino acids in all strains.

Our initial goal was to obtain intracellular concentrations for a comparative analysis. The challenge here was that cellular volume was highly heterogeneous in all strains under the salt condition (Fig. 2), hindering the intracellular concentration measurements that require defined cell volume. Therefore, we first normalized metabolite abundance in each strain by its cell dried weight (Fig. 5). The normalized metabolites abundance was compared between strains and conditions, as shown by the heatmap of $\log 2$ fold change in the Fig. 5 . From fixed weight of cell extracts, Zm6 cells without stress (Zm6 NS) generally comprised larger pools of 

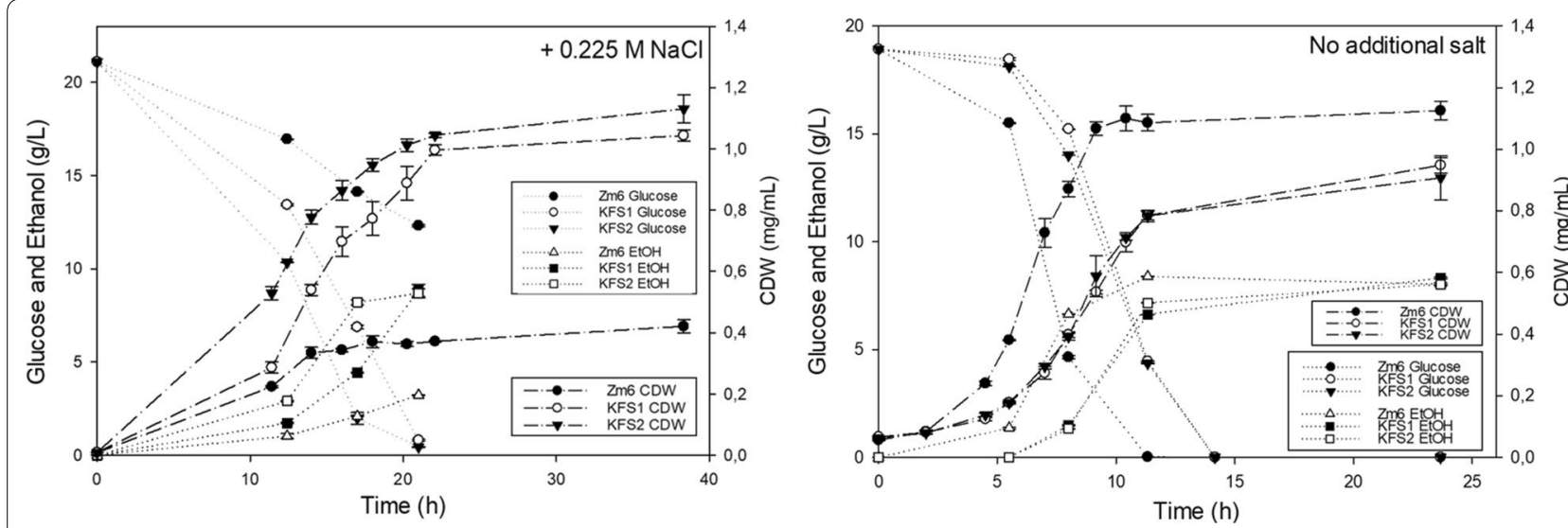

Fig. 3 Fermentation profiles of studied strains under salt or non-salt conditions. The plots show growth curve, glucose consumption and ethanol production under the saline $(0.225 \mathrm{M} \mathrm{NaCl})$ condition (left panel) or the non-salt condition (right). The error bars represent standard deviations of 3 independent measurements. The strains KFS1 and KFS2 exhibited improved growth and fermentation capacity under the saline condition, while these strains grew mildly slower than the parental strain did under the non-salt condition

the metabolites than $\mathrm{Zm6}$ cells under the salt condition (Zm6 S) (Fig. 5, left panel). Interestingly, several free amino acids including proline were the only metabolites found upregulated under the salt condition per fixed dry weight, although mildly. Similarly, the extracts from evolved strains under the salt condition (KFS1 S, KFS2 S) showed smaller pool sizes of the metabolites than from Zm6 NS (Fig. 5, middle and right panel).

Next, we stained the membrane of $Z$. mobilis cells with a staining dye Fm4-64 to observe if there was a compartmentalization within the cell. We observed no septum formation nor membrane organelle in the bulged $\mathrm{Zm6}$ cells (Fig. 6). Apparently, the salted Zm6 single cell volume was much larger than that of rod shape $\mathrm{Zm} 6$ cell under non-salt condition (Figs. 2, 6).

According to the previous studies, protein, DNA and RNA are the main components constituting about $70-80 \%$ of weight in bacterial cells [28]. It is less likely that slow growing filamentous cells $(\mathrm{Zm} 6 \mathrm{~S})$ possessed more dense macro molecules per fixed cell volume than actively growing small cells (Zm6 NS). We therefore speculated that $\mathrm{Zm} 6 \mathrm{~S}$ is expected to have larger cell volume per fixed amount of CDW than that of Zm6 NS. This further leads to that the $\log 2$ ratio of $\mathrm{Zm} 6 \mathrm{~S} / \mathrm{Zm} 6$ NS in the Fig. 5, normalized by CDW, should decrease when intracellular concentration is deployed as a unit, as Zm6 S cells most likely possessed larger cell volume per same amount of CDW, in comparison to Zm6 NS cells. Thus, our metabolomics data suggest that Zm6 cells did not drastically accumulate central metabolites or free amino acids under the salt condition. Most of the ED pathway metabolites and nucleosides in $\mathrm{Zm} 6 \mathrm{~S}$ was drastically downregulated, coinciding with low growth and slow glucose uptake. Moreover, osmoprotectant such as proline, was not found significantly accumulated either. Although, it was not completely excluded that atypical osmoprotectants that were not included in our targeted analysis might have been upregulated in $\mathrm{Zm} 6 \mathrm{~S}$ cells during the stress response.

The KFS1 and KFS2 cells under saline condition exhibited heterogeneous cell shapes, ranging from fragmented cells to long extended cells (Fig. 2). The membrane staining showed that the cells could produce a septum at several locations, unlike Zm6 cells. Nevertheless, KFS1 cells did not complete division and instead formed long filaments, and KFS1 S cell compartment size was found overall larger than that of Zm6 S (Fig. 6). Similar to the case of comparison of Zm6 extracts between saline conditions, bigger volume and slower growth of KFS1 S and KFS2 $\mathrm{S}$ to Zm6 NS implied that the actual intracellular concentration ratio of KFS1 or KFS2 S to Zm6 NS was smaller than the $\log 2$ ratio depicted in the Fig. 5 . This suggests that the evolved strains did not accumulate osmolytes to counter the stress either.

To further understand the resilient mechanism in evolved strains, we measured the intracellular ratio of reduced/oxidized form of NAD cofactor (NADH/ NAD) by an enzymatic assay. Malate dehydrogenase and oxoglutarate dehydrogenase complex are not encoded in the $Z$. mobilis genome [3, 29], which influences regeneration of NAD. Previous work suggested that maintaining a low NADH/NAD ratio appear to be important in the response against salt and acetic acid stress, to sustain glycolysis in the ED pathway that requires oxidized NAD $[15,30]$. To examine if the redox regulation of co-factor conferred the resilience, 

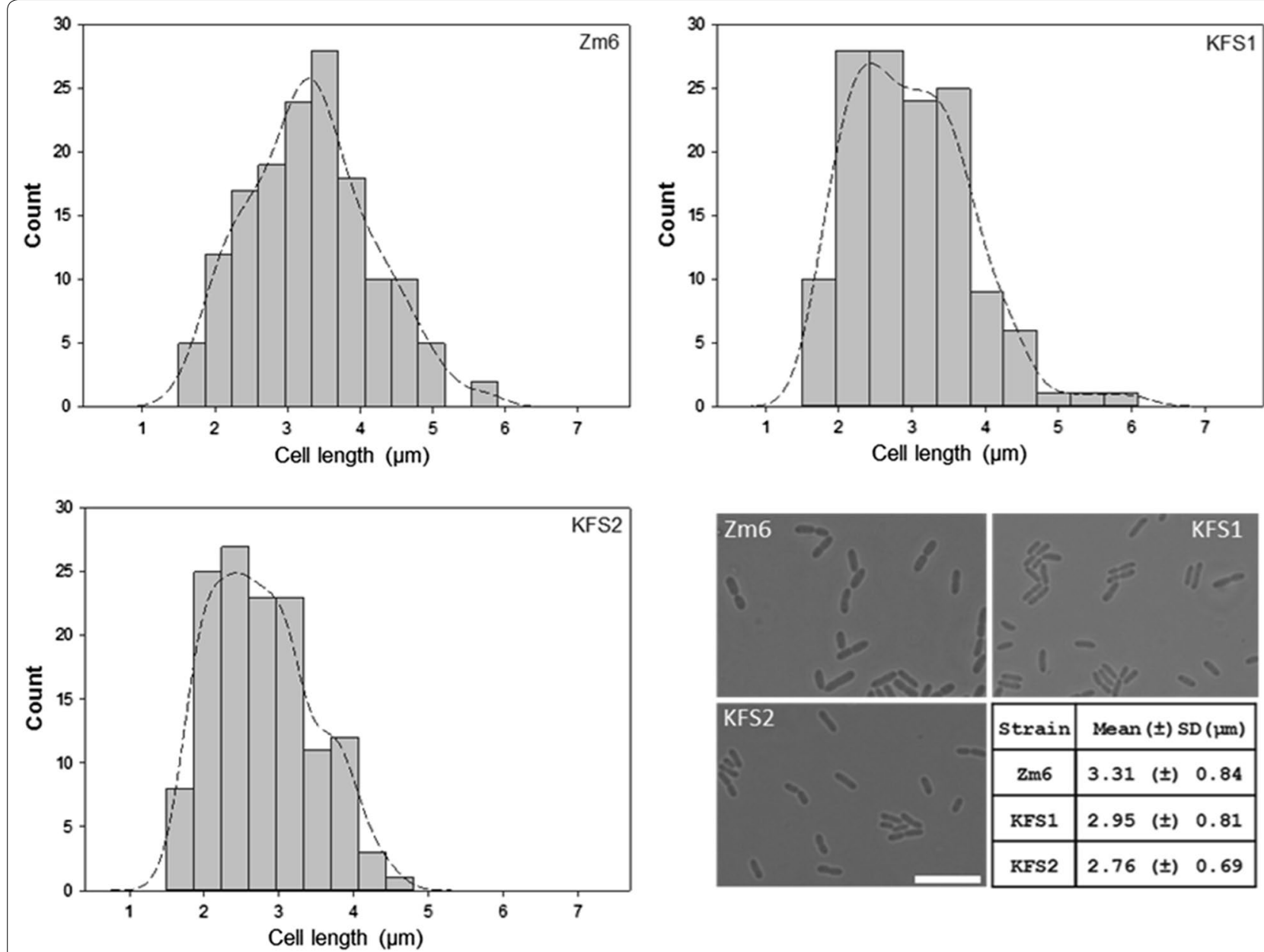

Fig. 4 Cell sizes are reduced in the evolved strains under non-saline conditions. Histograms (top two and left bottom panel) show the distribution of cellular length of growing Zm6 (top left), KFS1 (top right) and KFS2 (left bottom) under the non-saline condition ( $N=150$ for Zm6, N=133 for KFS1 and KFS2). The cells were grown in the complex medium under anaerobic condition until $\mathrm{OD}_{600}$ of the cultures reached around 1 in all strain. Cells were then mounted on the agarose-pad and imaged by a phase contrast microscope. Kernel density was plotted using bandwidth of 0.289 for all histograms. Phase contrast images of each strain under non-saline condition (left bottom, strain is indicated in the image) is shown with a scale bar $10 \mu \mathrm{m}$ applied for all images. Small table in the right bottom displays the mean value of cellular length ( $\mu \mathrm{m})$ in each strain. SD stands for standard deviation

we examined the NADH/NAD ratio in the strains. The analysis showed that saline stress increased the ratio in Zm6 (Table 1), as it was previously shown [15]. Interestingly, upregulation of the NADH/NAD ratio under saline conditions was also observed in evolved strains as well, within a similar range of shift to $\mathrm{Zm6}$. These data indicate that a modulation of NADH/NAD did not appear to play a major role in the evolved strains. Although the modulation of ratio is vital in general $Z$. mobilis stress response, as shown by previous studies $[15,30]$, the resilience to salt stress in the evolved appeared to be mediated by a separate mechanism.

\section{Identification of gene loci}

We examined the genomes of the evolved strains in an attempt to identify mutational changes that might be responsible for the observed stress tolerance phenotype. The whole genomes of parental and evolved strains were sequenced and aligned against the reference genome [29]. The analysis showed that our lab stock Zm6 strain possesses several mutations, 5 pointmutations and 2 frame shift-mutations of the ORFs in its genome (Additional file 1: Table S2). These mutations likely arose during our previous laboratory practices. We found several mutations only arose in the evolved 


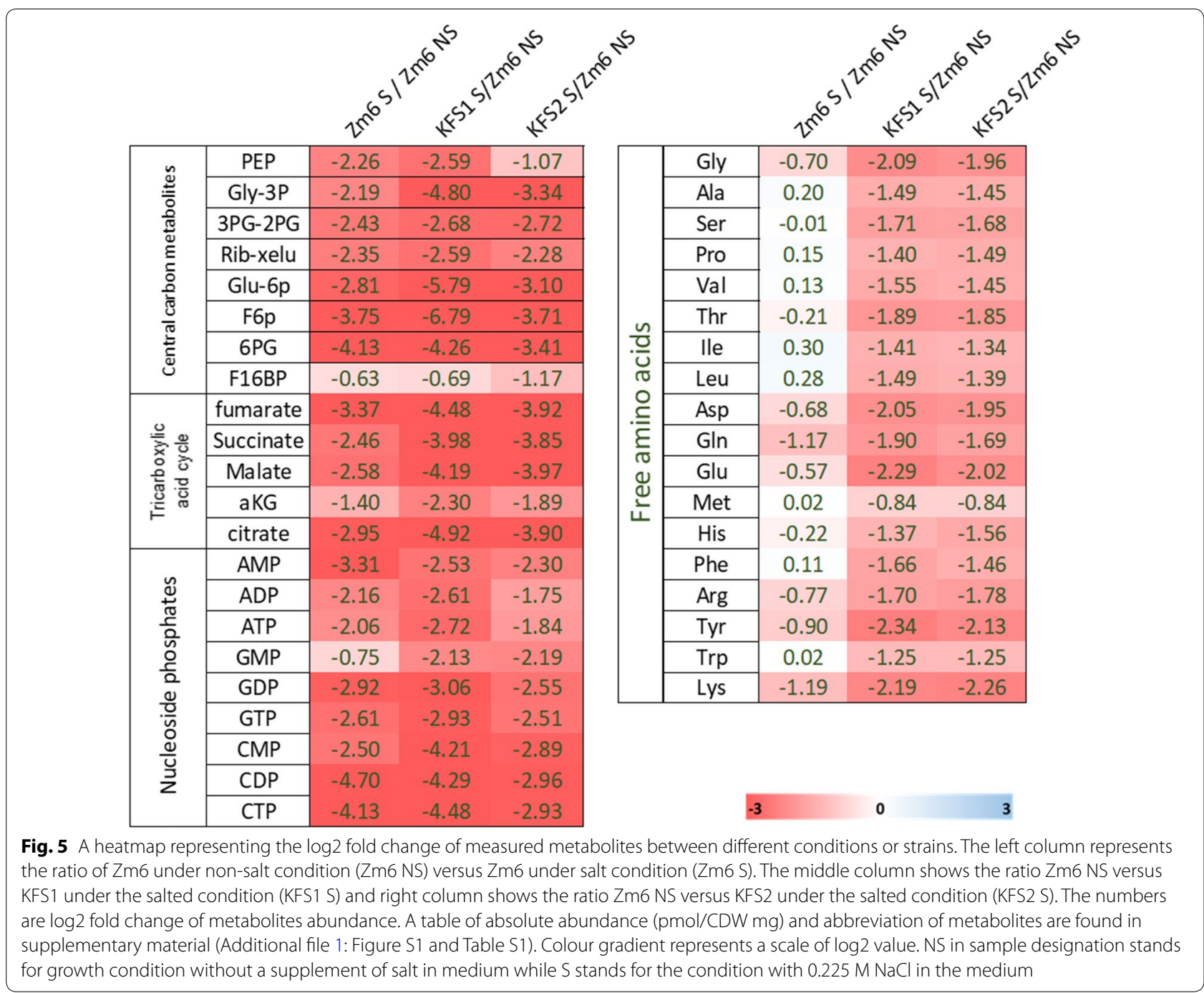

strains (Table 2). A disruptive insertion in ZZ6_1449 coding carboxyl-terminal protease (CTP) was among them. CTP is found in all kingdom of life and mainly cleaves serine or lysine nearby at $\mathrm{C}$-terminus of substrate. In bacteria, it has been shown that mutation in CTP caused alternation in cell envelop and higher sensitivity to antibiotics [31] and the osmotic down-shift in Escherichia coli [32]. In Pseudomonas aeruginosa, the disruption of CTP resulted in impaired growth in the medium with low salt [33]. Although, it was not clear if the observed phenotypes of low salt sensitivity in $E$. coli and $P$. aeruginosa were a direct consequence of an inactivation of the effector proteins of CTP that are involved in the osmo-protection, or, might be an indirect consequence of the altered PG metabolism caused by the disruption of CTP in the mutant [32, 33]. The reported phenotypes in $E$. coli and $P$. aeruginosa are to some extent consistent with our results from the growth profile. In addition, we found three other pointmutations only found in KFS1 and KFS2, which might be linked to the salt-resilient phenotypes. However, the function of these genes is not annotated. We found a point-mutation only found in KFS2, and an annotation of mutated gene is not available from the database. There are mild differences between KFS1 $\mathrm{S}$ and KFS2 $\mathrm{S}$ in morphology and growth, and it cannot be excluded that the mutation in the uncharacterized gene conferred extra resilience in KFS2 as well. Together with available literature, it suggests that disruption of ZZ6_1449 was mainly responsible for the improvement of growth. However, the genetic complementation test on the evolved strains, or, an introduction of disruptive mutation to $c t p$ locus in the parental $\mathrm{Zm} 6$ strain should be performed to prove the link between the CTP mutation and the improved phenotype. 


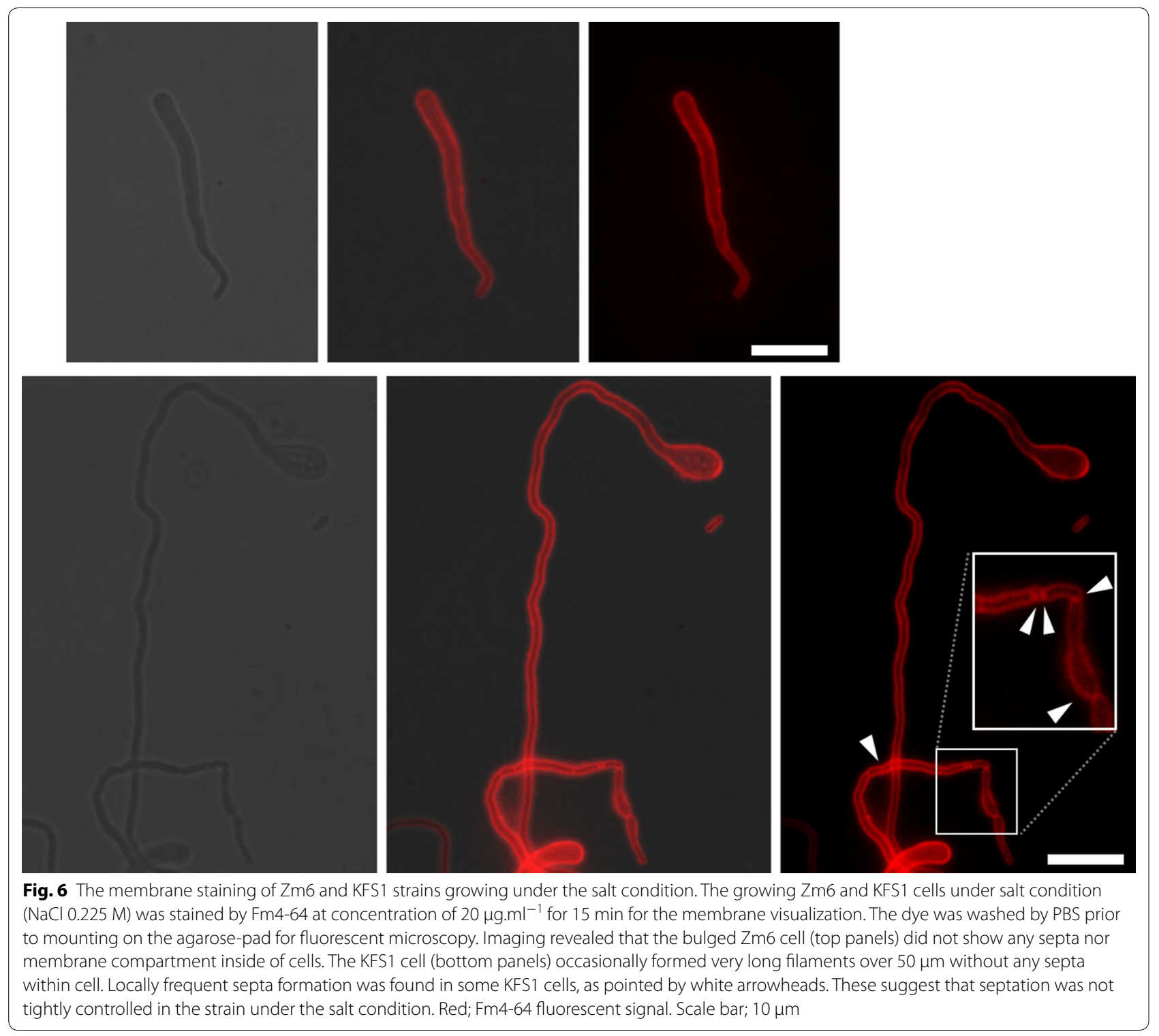

Table 1 The NADH/NAD ratio in growing Zm6 and evolved strains under the non-saline or the saline conditions

\begin{tabular}{lll}
\hline Strain & NADH/NAD $( \pm)$ SD & \\
\cline { 2 - 3 } & Non-salt condition & Salt condition \\
\hline Zm6 & $0.57( \pm) 0.03$ & $0.99( \pm) 0.11$ \\
KFS1 & $0.40( \pm) 0.05$ & $0.97( \pm) 0.20$ \\
KFS2 & $0.51( \pm) 0.15$ & $1.08( \pm) 0.15$ \\
\hline
\end{tabular}

\section{Conclusion}

The present study successfully generated strains that can grow and produce ethanol in added salt media usually inhibitory to wild type strains. Our approach was to bias selection by sedimentation of stressed filamentous cells, which might be utilized in other kinds of strain improvement in $Z$. mobilis. Interestingly, the evolved strains did not adapt to the saline environment by adjusting the prototypical osmolyte concentration or modulation of the intracellular NADH/NAD ratio. Comparative genome sequencing of wild type and evolved strains revealed that the disruption of $c t p$ was likely responsible for the improvement by altering cell envelope profile, yet an experimental evidence is required for confirming the disruption of CTP as a cause of the phenotype in the evolved strains. Further elucidation of the evolved strains might shed light on mechanistic understanding of salt stress response in Z. mobilis. 
Table 2 A list of mutations only found in the evolved strains

\begin{tabular}{llllll}
\hline Position & Detected sequence & Locus & Mutation & (annotated) Function & Strains \\
\hline 7181 & C $->$ T & ZZ6_0006 & A273T & Carboxymethylenebutenolidase & KFS1 and KFS2 \\
342703 & C $>$ T & ZZ6_0303 & G192R & Hypothetical protein & KFS1 and KFS2 \\
1641047 & A $>$ AGGCTCAGG & ZZ6_1449 & Insertion at L282, frame & Carboxyl-terminal protease & KFS1 and KFS2 \\
1650537 & ACCCATTGATTT & & shift & Hypothetical protein & KFS2 \\
\hline
\end{tabular}

\section{Materials and method}

\section{Adaptive laboratory evolution}

Z. mobilis ATCC 29191 was used as a parental strain for laboratory evolution. Zm6 was cultivated in a complex growth medium containing glucose $(20 \mathrm{~g} / \mathrm{L})$, yeast extract (5 g/L), $\mathrm{NH}_{4} \mathrm{SO}_{4}(1 \mathrm{~g} / \mathrm{L}), \mathrm{KH}_{2} \mathrm{PO}_{4}(1 \mathrm{~g} / \mathrm{L}), \mathrm{MgSO}_{4}$ $(0.5 \mathrm{~g} / \mathrm{L})$, with supplemental $\mathrm{NaCl}$ (final concentration; $0.2 \mathrm{M}-0.225 \mathrm{M})$ to generate a salt tolerant strain. The complex medium was flashed by nitrogen gas filtered through sterilized $0.2 \mu \mathrm{m}$ Supor $^{\circledR}$ (polyethersulfone) membrane (PALL) prior to use. A glycerol stock from a $-80{ }^{\circ} \mathrm{C}$-freezer was inoculated into the medium to make a starter culture. It should be noted that the glycerol stock was made from the overnight culture originated from a single colony of our Zm6 lab stock strain. $12 \mathrm{~mL}$ of anaerobic $\mathrm{Zm} 6$ culture was grown at $30{ }^{\circ} \mathrm{C}$ in a tightly capped $15 \mathrm{ml}$ falcon tube with shaking at $200 \mathrm{rpm} .30 \mu \mathrm{L}$ of inoculum was transferred from previous round of culture for evolution. Transfer of cells to fresh medium was performed when cells reached stationary phase. First 13 transfers were done in the medium with $0.2 \mathrm{M}$ salt, generating KFS1 strain. The rest of evolution was performed in the medium with $0.225 \mathrm{M}$ for another 8 transfers, generating KFS2 strain.

\section{Characterization of evolved strains}

To analyze cellular growth under the salt condition, an overnight culture was inoculated into anaerobic standing cultures and incubated at $30{ }^{\circ} \mathrm{C}$. It should be noted that the used inoculum was grown in the same growth medium without additional $\mathrm{NaCl}$, thus the inoculated cells were not adapted to the salt condition. The optical density (absorbance at $600 \mathrm{~nm}$ ) of culture was measured using a spectrophotometer (VWR). The cell dry weight was measured as follows; growing cells were centrifuged for $5 \mathrm{~min}(4500 \times g)$ and washed in phosphate buffered saline (PBS) solution (OXOID). The washed cells were transferred to a foil cup package (VWR) and dried in an oven at $90{ }^{\circ} \mathrm{C}$ till the solutions got completely dried. The dried cells on foil were weighed and subtracted by the tare weight of foil and dried PBS. Figure 3 was made using the measured $\mathrm{OD}_{600}$ and the conversion factors CDW $[\mathrm{g} / \mathrm{L}] / \mathrm{OD}_{600}$ (Additional file 1: Table S3).
Determination of glucose consumption and ethanol production in spent media was performed using Waters 2695e Alliance HPLC (Waters) with Hi-plex column $(300 \times 7.7 \mathrm{~mm}$, Agilent $)$ under a running condition; $0.05 \mathrm{M}$ sulfuric acid as mobile phase at a flow rate of $0.8 \mathrm{~mL} / \mathrm{min}$. MQuant ${ }^{\circledR}$ Glucose test kit (Merck) was also tested for an estimation of glucose presence (10-500 mg/L).

\section{Metabolomics}

Cell extractions were prepared as described in [34, 35]. Central carbon metabolites and nucleoside-phosphate were analyzed by capillary ion chromatography [34] coupled to tandem mass spectrometry, TQ-XS (Waters). Free amino acids were first derivatized by Edman's reagent [36]. Derivatized samples were analyzed by UPLC (waters) coupled to TQ-XS as described in [37]. All metabolites measurements were corrected by isotopic dilution method as described in [37].

Determination of the NADH/NAD ratio was performed using an enzymatic assay kit, following the instruction supplied by the manufacturer (Sigma). Briefly, growing culture was centrifuged $(3 \mathrm{~min}, 4500 \times g$ ) and the supernatant was carefully discarded. Pellets were quickly frozen by liquid nitrogen for storage at $-80^{\circ} \mathrm{C}$. Upon the measurements, the frozen cells were thawed on ice and resuspended in the extraction buffer supplied from the kit. An absorbance at $450 \mathrm{~nm}$ was measured every $20 \mathrm{~min}$ for $4 \mathrm{~h}$ using 96 well-plates in a plate-reader spark $20 \mathrm{M}$ (Tecan).

\section{Fluorescent microscopy and image analysis}

Live Z. mobilis cells were observed using a Zeiss Axio Imager Z2 microscope. The images were captured by Axiocam MR R3 (ZEISS) and analyzed by ZEN 2.3 pro software (ZEISS). For staining membrane, growing cultures were centrifuged $(4500 \mathrm{xg})$ for $5 \mathrm{~min}$ and the pellet was resuspended in phosphate buffered saline solution. FM464 (Thermo Fisher Scientific) was then added at a final concentration of $\left(20 \mu \mathrm{g} \cdot \mathrm{ml}^{-1}\right)$ and incubated for $15 \mathrm{~min}$. Cells were then washed in PBS again and mounted on PBS agarose-pad $(1 \% \mathrm{w} / \mathrm{v})$. Comparison of cell size was 
done using the phase contrast images and the scale bars obtained from the imaging.

\section{Whole genome sequencing}

The whole genome of the studied strains was sequenced by GATC re-sequencing service (INVIEW Genome sequencing). Total DNA extraction was performed combining a lysing method [38] and the D-neasy blood tissue kit (Qiagen). Briefly, a lysozyme treatment was performed as follows. An overnight culture was centrifuged $(20,000 \times g)$ for $1 \mathrm{~min}$, and the pellets were washed in TE buffer (10 mM Tris-Cl pH7.5, 1 mM EDTA) and centrifuged again. The pellets were resuspended in a lysozyme solution and incubated for $30 \mathrm{~min}$ at $37{ }^{\circ} \mathrm{C}$. (Lysozyme solution; lysozyme $50 \mathrm{mg} \cdot \mathrm{ml}^{-1}$ in buffer containing $10 \mathrm{mM}$ Tris-Cl pH 8, $2.5 \mathrm{mM}$ EDTA, $20 \mathrm{mM} \mathrm{NaCl}$ ). This procedure was followed by a proteinase $\mathrm{K}$ incubation $\left(20 \mathrm{mg} \cdot \mathrm{ml}^{-1}, 1 \mathrm{~h}\right.$ at $\left.50^{\circ} \mathrm{C}\right)$ and the rest of procedures followed the instructions provided by the manufacturer. A concentration and purity of genomic DNA was determined using nanodrop one (thermo scientific).

\section{Supplementary information}

Supplementary information accompanies this paper at https://doi. org/10.1186/s12934-020-01406-0.

Additional file 1: Figure S1. Metabolite abundance [pmol.mg ${ }^{-1}$ (CDW)] in each strain was measured by mass spectrometry-based metabolomics. The data was used for $\log 2$ fold ratio heatmap in the Fig. 5. Table S1. A list of abbreviation of measured metabolites. Table S2. A list of detected mutations in the lab stock Zm6 strain. Table S3. The CDW/OD 600 conversion factor in each strain under saline/non-saline conditions.

\section{Abbreviations}

ED: Entner-Doudoroff; NAD: Nicotinamide adenine dinucleotide; CDW: Cell dry weight; OD: Optical density; HPLC: High pressure liquid chromatography; UPLC: Ultra-performance liquid chromatography; SD: Standard deviation; WGS: Whole genome sequencing.

\section{Acknowledgements}

The authors thank Dr. Kåre Andre Kristiansen for an excellent technical assistance. The authors would like to thank the anonymous reviewer for valuable comments and corrections to the manuscript.

\section{Authors' contributions}

KF conceptualized and designed the study, conducted the experiments, interpreted and analyzed the data, and wrote and edited the draft manuscript. PB supervised the study, interpreted and analyzed the data, and edited the draft manuscript. Both authors read and approved the final manuscript.

\section{Funding}

KF and PB was financially supported by the Research Council of Norway, Grant Number 258657

\section{Availability of data and materials}

The datasets and strains generated from the present study are available from the corresponding author on a reasonable request.

Ethics approval and consent to participate

Not applicable.
Consent for publication

Not applicable.

\section{Competing interests}

The authors declare that they have no competing interests.

Received: 25 April 2020 Accepted: 13 July 2020

Published online: 20 July 2020

\section{References}

1. Turner TL, Kim H, Kong II, Liu J-J, Zhang G-C, Jin Y-S. Engineering and evolution of Saccharomyces cerevisiae to produce biofuels and chemicals. In: Zhao H, Zeng A-P, editors. Synthetic Biology-Metabolic Engineering. Cham: Springer International Publishing; 2018. p. 175-215.

2. Weisser P, Krämer R, Sahm H, Sprenger GA. Functional expression of the glucose transporter of Zymomonas mobilis leads to restoration of glucose and fructose uptake in Escherichia coli mutants and provides evidence for its facilitator action. J Bacteriol. 1995:177(11):3351.

3. Seo J-S, Chong H, Park HS, Yoon K-O, Jung C, Kim JJ, Hong JH, Kim H, Kim $\mathrm{J}-\mathrm{H}, \mathrm{Kil} \mathrm{J}-\mathrm{I}$, et al. The genome sequence of the ethanologenic bacterium Zymomonas mobilis ZM4. Nat Biotechnol. 2005;23(1):63-8.

4. Kalnenieks U: Physiology of Zymomonas mobilis: Some Unanswered Questions. In: Advances in Microbial Physiology. Edited by Poole RK, vol. 51: Academic Press; 2006: 73-117.

5. Kremer TA, LaSarre B, Posto AL, McKinlay JB. N2 gas is an effective fertilizer for bioethanol production by Zymomonas mobilis. Proc Natl Acad Sci. 2015:112(7):2222-6

6. Yang S, Fei Q, Zhang Y, Contreras LM, Utturkar SM, Brown SD, Himmel ME, Zhang M. Zymomonas mobilis as a model system for production of biofuels and biochemicals. Microb Biotechnol. 2016;9(6):699-717.

7. Rogers PL, Jeon YJ, Lee KJ, Lawford HG: Zymomonas mobilis for Fuel Ethanol and HigherValue Products. In: Biofuels. Edited by Olsson L. Berlin, Heidelberg: Springer Berlin Heidelberg: 2007: 263-288.

8. Kalnenieks U, Balodite E, Strähler S, Strazdina I, Rex J, Pentjuss A, Fuchino K, Bruheim P, Rutkis R, Pappas KM, et al. Improvement of acetaldehyde production in Zymomonas mobilis by engineering of its aerobic metabolism. Front Microbiol. 2019;10:2533.

9. He MX, Wu B, Qin H, Ruan ZY, Tan FR, Wang JL, Shui ZX, Dai LC, Zhu QL, Pan K, et al. Zymomonas mobilis: a novel platform for future biorefineries. Biotechnol Biofuels. 2014;7(1):101.

10. Swings J, De Ley J. The biology of Zymomonas. Bacteriol Rev. 1977:41(1):1-46.

11. Vriesekoop F, Rasmusson M, Pamment NB. Respective effects of sodium and chloride ions on filament formation and growth and ethanol production in Zymomonas mobilis fermentations. Lett Appl Microbiol. 2002;35(1):27-31.

12. Franden MA, Pilath HM, Mohagheghi A, Pienkos PT, Zhang M. Inhibition of growth of Zymomonas mobilis by model compounds found in lignocellulosic hydrolysates. Biotechnol Biofuels. 2013;6(1):99-99.

13. Wang J-L, Wu B, Qin H, You Y, Liu S, Shui Z-X, Tan F-R, Wang Y-W, Zhu Q-L, $\mathrm{Li} Y$-B, et al. Engineered Zymomonas mobilis for salt tolerance using EZ-Tn5-based transposon insertion mutagenesis system. Microb Cell Fact. 2016:15(1):101.

14. Gao X, Gao Q, Bao J. Improving cellulosic ethanol fermentability of Zymomonas mobilis by overexpression of sodium ion tolerance gene ZMO0119. J Biotechnol. 2018;282:32-7.

15. Hayashi T, Kato T, Watakabe S, Song W, Aikawa S, Furukawa K. The respiratory chain provides salt stress tolerance by maintaining a low NADH/ NAD + ratio in Zymomonas mobilis. Microbiology. 2015;161(12):2384-94.

16. Sleator RD, Hill C. Bacterial osmoadaptation: the role of osmolytes in bacterial stress and virulence. FEMS Microbiol Rev. 2002;26(1):49-71.

17. Sévin DC, Stählin JN, Pollak GR, Kuehne A, Sauer U. Global Metabolic Responses to Salt Stress in Fifteen Species. PLoS ONE. 2016:11(2):e0148888.

18. Kohler C, Lourenço RF, Bernhardt J, Albrecht D, Schüler J, Hecker M, Gomes SL. A comprehensive genomic, transcriptomic and proteomic analysis of a hyperosmotic stress sensitive a-proteobacterium. BMC Microbiol. 2015;15(1):71. 
19. Sootsuwan K, Thanonkeo P, Keeratirakha N, Thanonkeo S, Jaisil P, Yamada M. Sorbitol required for cell growth and ethanol production by Zymomonas mobilis under heat, ethanol, and osmotic stresses. Biotechnol Biofuels. 2013;6(1):180.

20. Huang S, Xue T, Wang Z, Ma Y, He X, Hong J, Zou S, Song H, Zhang M. Furfural-tolerant Zymomonas mobilis derived from error-prone PCR-based whole genome shuffling and their tolerant mechanism. Appl Microbiol Biotechnol. 2018;102(7):3337-47.

21. Mohagheghi A, Linger JG, Yang S, Smith H, Dowe $N$, Zhang M, Pienkos PT. Improving a recombinant Zymomonas mobilis strain $8 \mathrm{~b}$ through continuous adaptation on dilute acid pretreated corn stover hydrolysate. Biotechnol Biofuels. 2015;8(1):55.

22. Shui ZX, Qin H, Wu B, Ruan Z, Wang L, Tan FR, Wang JL, Tang XY, Dai LC, Hu GQ, et al. Adaptive laboratory evolution of ethanologenic Zymomonas mobilis strain tolerant to furfural and acetic acid inhibitors. Appl Microbiol Biotechnol. 2015;99(13):5739-48.

23. Liu Y-F, Hsieh C-W, Chang Y-S, Wung B-S. Effect of acetic acid on ethanol production by Zymomonas mobilis mutant strains through continuous adaptation. BMC Biotechnol. 2017;17(1):63.

24. Wang W, Wu B, Qin H, Liu P, Qin Y, Duan G, Hu G, He M. Genome shuffling enhances stress tolerance of Zymomonas mobilis to two inhibitors. Biotechnol Biofuels. 2019;12(1):288.

25. Jones-Burrage SE, Kremer TA, McKinlay JB. Cell Aggregation and aerobic respiration are important for Zymomonas mobilis ZM4 survival in an aerobic minimal medium. Appl Environ Microbiol. 2019;85(10):e00193-00119.

26. Hayashi T, Kato T, Furukawa K. Respiratory chain analysis of Zymomonas mobilis mutants producing high levels of ethanol. Appl Environ Microbiol. 2012;78(16):5622-9.

27. Krämer R. Bacterial stimulus perception and signal transduction: response to osmotic stress. Chem Record. 2010;10(4):217-29.

28. Schönheit P, Buckel W, Martin WF. On the Origin of Heterotrophy. Trends Microbiol. 2016;24(1):12-25.

29. Desiniotis A, Kouvelis VN, Davenport K, Bruce D, Detter C, Tapia R, Han C, Goodwin LA, Woyke T, Kyrpides NC, et al. Complete genome sequence of the ethanol-producing Zymomonas mobilis subsp. mobilis Centrotype ATCC 29191. J Bacteriol. 2012;194(21):5966.
30. Wu B, Qin H, Yang Y, Duan G, Yang S, Xin F, Zhao C, Shao H, Wang Y, Zhu Q, et al. Engineered Zymomonas mobilis tolerant to acetic acid and low pH via multiplex atmospheric and room temperature plasma mutagenesis. Biotechnol Biofuels. 2019;12(1):10.

31. Seoane A, Sabbaj A, McMurry LM, Levy SB. Multiple antibiotic susceptibility associated with inactivation of the prc gene. J Bacteriol. 1992;174(23):7844-7.

32. Hara H, Yamamoto Y, Higashitani A, Suzuki H, Nishimura Y. Cloning, mapping, and characterization of the Escherichia coli prc gene, which is involved in C-terminal processing of penicillin-binding protein 3. J Bacteriol. 1991;173(15):4799.

33. Srivastava D, Seo J, Rimal B, Kim SJ, Zhen S, Darwin AJ. A proteolytic complex targets multiple cell wall hydrolases in Pseudomonas aeruginosa. mBio. 2018;9(4):e00972-00918.

34. Kvitvang HFN, Kristiansen KA, Bruheim P. Assessment of capillary anion exchange ion chromatography tandem mass spectrometry for the quantitative profiling of the phosphometabolome and organic acids in biological extracts. J Chromatogr A. 2014;1370:70-9.

35. Fuchino K, Kalnenieks U, Rutkis R, Grube M, Bruheim P. Metabolic profiling of glucose-fed metabolically active resting Zymomonas mobilis Strains. Metabolites. 2020;10(3):81.

36. Cohen SA, Strydom DJ. Amino acid analysis utilizing phenylisothiocyanate derivatives. Anal Biochem. 1988;174(1):1-16.

37. Røst LM, Brekke Thorfinnsdottir L, Kumar K, Fuchino K, Eide Langørgen I, Bartosova Z, Kristiansen KA, Bruheim P. Absolute quantification of the central carbon metabolome in eight commonly applied prokaryotic and eukaryotic model systems. Metabolites. 2020;10(2):74.

38. Gill C, van de Wijgert JHHM, Blow F, Darby AC. Evaluation of Lysis Methods for the Extraction of Bacterial DNA for Analysis of the Vaginal Microbiota. PLoS ONE. 2016;11(9):e0163148.

\section{Publisher's Note}

Springer Nature remains neutral with regard to jurisdictional claims in published maps and institutional affiliations.
Ready to submit your research? Choose BMC and benefit from:

- fast, convenient online submission

- thorough peer review by experienced researchers in your field

- rapid publication on acceptance

- support for research data, including large and complex data types

- gold Open Access which fosters wider collaboration and increased citations

- maximum visibility for your research: over $100 \mathrm{M}$ website views per year

At BMC, research is always in progress.

Learn more biomedcentral.com/submissions 